\title{
Effect of (3-aminopropyl)trimethoxysilane on mechanical properties of PLA/PBAT blend reinforced kenaf fiber
}

\begin{abstract}
The composite-based poly(lactic acid) (PLA)/poly(butylene adipate-co-terephthalate) (PBAT)/kenaf fiber has been prepared using melt blending method. A PLA/PBAT blend with the ratio of 90:10 wt\%, and the same blend ratio reinforced with various amounts of kenaf fiber have been prepared and characterized. However, the addition of kenaf fiber has reduced the mechanical properties sharply due to the poor interaction between the fiber and polymer matrix. Modification of the composite by (3-aminopropyl)trimethoxysilane (APTMS) showed improvements in mechanical properties, increasing up to 42.46, 62.71 and $22.00 \%$ for tensile strength, flexural strength and impact strength, respectively. The composite treated with $2 \%$ APTMS successfully exhibited optimum tensile strength (52.27 MPa), flexural strength $(64.27 \mathrm{MPa})$ and impact strength $(234.21 \mathrm{~J} / \mathrm{m})$. Morphological interpretation through scanning electron microscopy (SEM) reveals improved interaction and interfacial adhesion between PLA/PBAT blend and kenaf fiber. The fiber was well distributed and remained in the PLA/PBAT blend evenly. DMA results showed lower storage modulus ( $\left.E^{\prime}\right)$ for PLA/PBAT/kenaf fiber blend and an increase after modification by 2 wt $\%$ APTMS. Conversely, the relative damping properties decreased. Based on overall results, APTMS can be used as coupling agent for the composite since APTMS can improve the interaction between hydrophilic natural fibers and non-polar polymers.
\end{abstract}

Keyword: APTMS; Biocomposites; Coupling agent; Mechanical properties; Melt blending. 